\title{
Eptinezumab treatment initiated during a migraine attack is associated with meaningful improvement in patient- reported outcome measures: secondary results from the randomized controlled RELIEF study
}

Peter McAllister ${ }^{1}$, Paul K. Winner ${ }^{2,3,4,6,6}$, Jessica Ailani ${ }^{7}$, Dawn C. Buse ${ }^{8,9}$, Richard B. Lipton ${ }^{8}$, George Chakhava ${ }^{10}$, Mette Krog Josiassen ${ }^{11}$, Annika Lindsten ${ }^{11}$, Lahar Mehta ${ }^{12}$, Anders Ettrup ${ }^{11}$ and Roger Cady ${ }^{13^{*}}$

\begin{abstract}
Background: Demonstrating therapeutic value from the patient perspective is important in patient-centered migraine management. The objective of this study was to investigate the impact of eptinezumab, a preventive migraine treatment, on patient-reported headache impact, acute medication optimization, and perception of disease change when initiated during a migraine attack.

Methods: RELIEF was a randomized, double-blind, placebo-controlled trial conducted between 2019 and 2020 in adults with $\geq 1$-year history of migraine and 4-15 migraine days per month in the 3 months prior to screening. Patients were randomized (1:1) to a 30-min infusion of eptinezumab $100 \mathrm{mg}$ or placebo within 1-6 h of a qualifying migraine attack onset. The 6-item Headache Impact Test (HIT-6) and 6-item Migraine Treatment Optimization Questionnaire (mTOQ-6) were administered at baseline and week 4, and the Patient Global Impression of Change (PGIC) at week 4. A post hoc analysis of these measures was conducted in patients who reported headache pain freedom at $2 \mathrm{~h}$ after infusion start.
\end{abstract}

\footnotetext{
* Correspondence: rcady@rkconsults.com

${ }^{13}$ Lundbeck LLC, Deerfield, IL, USA

Full list of author information is available at the end of the article
}

C C The Author(s). 2021 Open Access This article is licensed under a Creative Commons Attribution 4.0 International License, which permits use, sharing, adaptation, distribution and reproduction in any medium or format, as long as you give appropriate credit to the original author(s) and the source, provide a link to the Creative Commons licence, and indicate if changes were made. The images or other third party material in this article are included in the article's Creative Commons licence, unless indicated otherwise in a credit line to the material. If material is not included in the article's Creative Commons licence and your intended use is not permitted by statutory regulation or exceeds the permitted use, you will need to obtain permission directly from the copyright holder. To view a copy of this licence, visit http://creativecommons.org/licenses/by/4.0/ The Creative Commons Public Domain Dedication waiver (http://creativecommons.org/publicdomain/zero/1.0/) applies to the data made available in this article, unless otherwise stated in a credit line to the data. 
Results: Of 480 patients enrolled and treated, 476 completed the study and are included in this analysis. Mean baseline HIT-6 total scores indicated severe headache impact (eptinezumab, 65.1; placebo, 64.8). At week 4, the eptinezumab-treated group demonstrated clinically meaningful improvement in HIT-6 total score compared with placebo (mean change from baseline: eptinezumab, - 8.7; placebo, - 4.5; mean [95\% Cl] difference from placebo: $4.2[-5.75,-2.63], P<.0001)$, with greater reductions in each item score vs placebo $(P<.001$ all comparisons). Change in HIT-6 total score in the subgroup with 2-h headache pain freedom was - 13.8 for the eptinezumab group compared with -4.9 for the placebo group. mTOQ-6 total score mean change from baseline favored eptinezumab (change, 2.1) compared with placebo (1.2; mean [95\% Cl] difference: $0.9[0.3,1.5], P<.01)$. More eptinezumab-treated patients rated PGIC as much or very much improved than placebo patients (59.3\% vs 25.9\%).

Conclusions: When administered during a migraine attack, eptinezumab significantly improved patient-reported outcomes after 4 weeks compared with placebo, with particularly pronounced effects in patients reporting headache pain freedom at $2 \mathrm{~h}$ after infusion start.

Trial registration: ClinicalTrials.gov Identifier: NCT04152083. November 5, 2019.

Keywords: Migraine, Eptinezumab, CGRP, MBS

\section{Background}

Migraine is a highly prevalent neurological disease characterized by attacks of moderate to severe headache associated with physiological disruptions of neurological, gastrointestinal, and sensory function [1]. Migraine-related disability impacts not only the individual with migraine, but their families, workplace, and the healthcare system [2-5]. In addition, migraine has negative impact relating to missing important events, commitment avoidance, and feelings of guilt regarding the effect on family [6, 7]. Migraine is more common in women and most prevalent between the ages of 20-40 years, amplifying its impact on family and career development $[3,8]$. As migraine frequency increases, so does its impact on functionality, overall disability, and productivity [5, 9]. Patients with migraine balance lifestyle changes and trigger management with acute and preventive treatments taken to stop an attack or reduce the overall frequency of attacks [10-12]. In clinical trials for acute and preventive treatment of migraine, various endpoints and patient-reported outcome measures (PROMs) are used to evaluate efficacy [13, 14].

Eptinezumab, a humanized monoclonal antibody, is approved for the prevention of migraine in adults [15]. As an intravenous (IV) infusion with $100 \%$ bioavailability, eptinezumab has a $\mathrm{T}_{\max }$ of $30 \mathrm{~min}$ with rapid, high-affinity binding to calcitonin gene-related peptide [16]. In phase 3 studies in patients with episodic migraine [17] and chronic migraine [18], eptinezumab $100 \mathrm{mg}$ and $300 \mathrm{mg}$ met the primary efficacy endpoint, significantly reducing mean monthly migraine days (MMDs) over weeks 1-12. In RELIEF, a phase 3 clinical trial in patients with migraine, eptinezumab $100 \mathrm{mg}$ demonstrated efficacy in relieving headache pain and migraine-associated symptoms within 2 $\mathrm{h}$ of infusion start and reducing acute medication use compared with placebo when administered during a migraine attack [19].
The objective of this secondary analysis was to evaluate the impact of treatment in patients from the RELIEF study on PROMs captured 4 weeks after eptinezumab treatment initiated during a migraine attack.

\section{Methods \\ Study design and patients}

The RELIEF study (NCT04152083), including detailed methods, was previously described [19]. This was a 4week, phase 3, parallel-group, double-blind, placebocontrolled clinical trial in which patients received IV eptinezumab $100 \mathrm{mg}$ or placebo over $30 \mathrm{~min}$ within 1-6 $\mathrm{h}$ of a qualifying migraine attack (day 0 ). The use of rescue medication (any acute medication to treat migraine or migraine-associated symptoms) was not permitted in the 24-h period prior to receiving study treatment or within $2 \mathrm{~h}$ of infusion start. Only after $2 \mathrm{~h}$ post infusion start were patients permitted to use rescue medication.

RELIEF was conducted between November 2019 and July 2020 at 42 sites in the United States and 5 sites in the country of Georgia. The study was approved by a centralized institutional review board (or independent ethics committee at each study site, if required), with written informed consent obtained for each participant prior to the study's initiation.

Eligible patients were $18-75$ years of age with $\geq 1$-year history of migraine (defined by the International Classification of Headache Disorders, 3rd edition [ICHD-3] criteria) [1], with onset of first migraine $\leq 50$ years of age. All patients were required to have experienced migraine on 4-15 days per month in the 3 months prior to screening (screening occurred up to 8 weeks before dosing) to ensure that only patients eligible for preventive treatment were enrolled. Patients were required to have typical migraine attacks (4-72 h untreated), with headache pain of moderate to severe intensity, migraine- 
associated features, and a most bothersome symptom (MBS) of nausea, photophobia, or phonophobia. Additionally, patients were required to have a history of active or previous triptan use for migraine to help ensure a migraine diagnosis. Patients could not receive any monoclonal antibody treatment for any reason within 6 months prior to screening or any experimental, unregistered therapy within 30 days or 5 plasma half-lives (whichever was longer) prior to screening.

\section{Patient-reported outcome measures}

This report focuses on the 6-item Headache Impact Test (HIT-6) [20],6-item Migraine Treatment Optimization Questionnaire (mTOQ-6) [21], and Patient Global Impression of Change (PGIC) [22]. HIT-6 and mTOQ-6 outcomes were captured at the screening visit and 4 weeks after infusion, with PGIC captured at week 4 . Site staff reviewed questionnaires for completeness and clarity, and asked patients to complete any unanswered questions prior to patients leaving the clinic; the HIT-6 or mTOQ-6 total score was treated as missing if a patient response was missing the answer to $\geq 1$ questions.

HIT-6, as detailed in Additional file 1, Table 1, measures the impact on the ability to function normally in daily life when a headache occurs [20]. It is a 6-question, Likert-type, self-reporting questionnaire with each question scored as never $=6$, rarely $=8$, sometimes $=10$, very often $=11$, and always $=13$. The HIT- 6 total score is calculated from summing individual items (score range of 36-78 points), with score ranges representing the following burdens of migraine: severe impact $=\geq 60$, substantial impact $=56-59$, some impact $=50-55$, and little to no impact $=\leq 49$.

The mTOQ- 6 assesses the optimization of acute treatment in persons with migraine [21]. The MTOQ- 6 is a 6-question, Likert-type, self-reporting questionnaire, detailed in Additional file 1, Table 1, with each item scored as never $=1$, rarely $=2$, less than half the time $=3$, or half the time or more $=4$. The mTOQ- 6 total score is calculated by summing the score for each individual question (score range of 6-24 points), with a higher score indicating better treatment optimization.

The PGIC includes a single question concerning the subject's impression of the change in the severity of their illness since the start of the study [22]. Patients were asked, "Since receiving study drug in this study, how would you describe the change (if any) in activity limitations, symptoms, emotions, and overall quality of life as related to your migraine?" Answers were categorized into one of 7 categories: "very much improved", "much improved", "minimally improved", "no change", "minimally worse", "much worse", or "very much worse". At the week 4 visit, patients were asked to review brief instructions and then complete the assessment.

\section{Statistical analyses}

For the HIT-6 and mTOQ-6, item and total scores were summarized by treatment group at screening and week 4 visits and change from baseline to the week 4 visit was calculated. An analysis of covariance (ANCOVA) model was used to test for a difference between treatment arms for the total score, with the model using change from baseline at week 4 as the response variable. Baseline value, treatment group, and the stratification variables of concomitant preventive migraine treatment (use vs no use) and region (North America vs Georgia) were the independent variables. A similar ANCOVA was fitted for each item of the HIT- 6 and mTOQ- 6 . In the current analyses, the value from the week 4 visit was used for both outcome measures, regardless of whether it was an actual week 4 assessment or an early-termination assessment. The frequency distribution of PGIC responses at the week 4 visit was summarized descriptively.

An exploratory, post hoc analysis was undertaken to evaluate the ability of treatment to provide a clinically meaningful change in HIT-6 total score and item scores. A clinically meaningful within-person improvement in HIT-6 total score was defined as a 5-point or greater decrease, in line with the 2019 American Headache Society (AHS) position statement [23]. A further analysis based on a categorical HIT-6 analysis in patients with chronic migraine was also conducted [24], where a clinically meaningful within-person improvement was defined as $a \geq 6$-point decrease in HIT-6 total score, a $\geq 1$-category decrease in items $1-3$, and $a \geq 2$-category decrease in items 4-6.

As part of the efficacy assessment reported in Winner et al. [19], key secondary endpoints included headache pain freedom and absence of MBS at $2 \mathrm{~h}$ after infusion start, and additional secondary endpoints included headache pain freedom and absence of MBS at $4 \mathrm{~h}$ after infusion start. To evaluate the potential clinical meaningfulness of these improvements for changes in PROMs, a post hoc subgroup analysis of HIT-6 total score was conducted in patients who did and did not achieve these secondary endpoints. In addition to evaluating HIT-6 total score, the percentage of patients reporting a new migraine within the study period was calculated in each subgroup; the incidence of a new migraine was captured in an electronic diary from day 3 following treatment until a new migraine was reported (up to day 28).

\section{Results}

Patients

Of the 480 patients randomized to treatment, 235/238

(98.7\%) patients assigned to eptinezumab and 241/242

(99.6\%) patients assigned to placebo completed the study. Baseline demographics and characteristics have 
been previously reported [19], with similarity between eptinezumab and placebo groups (Table 1).

\section{6-item Headache Impact Test}

At baseline, the mean HIT-6 total score was 64.9 and was similar across treatment groups (eptinezumab, 65.1; placebo, 64.8). At week 4, the least squares (LS) mean (95\% CI) change from baseline in HIT-6 total score was $-8.7(-10.1,-7.3)$ with eptinezumab $100 \mathrm{mg}$ vs -4.5 $(-5.9,-3.1)$ with placebo $(P<.0001)$ (Table 2 and Fig. 1A). Using the 5-point reduction threshold [23] to determine a clinically meaningful change in HIT- 6 total score, a clinical response was achieved by $54.0 \%(n=$ 122) of patients treated with eptinezumab compared with $31.5 \%(n=73)$ of patients treated with placebo $(P<.0001)$ (Fig. 1B). When analyzed using the 6-point reduction threshold [24], a clinically meaningful change in HIT- 6 total score was achieved by $48.2 \%(n=109)$ of eptinezumab-treated patients compared with $28.9 \%$ ( $n=$ $67)$ of placebo patients $(P<.0001)$ (Fig. 1B).

At baseline, HIT-6 item scores were similar across treatment arms (see Additional file 1, Table 2) and reflective of a population with frequent migraine attacks [25]. The LS mean change from baseline at week 4 for HIT-6 items 1-3 was approximately -1.0 with eptinezumab, compared with approximately -0.5 with placebo across items $(P<.001$, all items $1-3$; Fig. $1 C)$. In the analysis of HIT-6 items 4-6, the LS mean change from baseline at week 4 ranged from -1.7 to -2.1 with eptinezumab and from -0.8 to -1.2 with placebo $(P<.0001$, all items 4-6; Fig. $1 C)$. For each HIT-6 item, $30 \%-40 \%$ of eptinezumab-treated patients achieved a clinical response; for patients who received placebo, $26.7 \%-33.2 \%$ achieved response on items $1-3$ and $15.1 \%-18.1 \%$ achieved response on items $4-6$ (Fig. 1D) $(P<.05$ for all except item 2 [limits daily activities]).

In patients reporting headache pain freedom at $2 \mathrm{~h}$, the mean HIT- 6 total score at baseline was 67.3 for the eptinezumab group $(n=54)$ and 64.5 for the placebo group $(n=29)$. At week 4 , the mean change from baseline in HIT-6 total score $(95 \% \mathrm{CI})$ in the eptinezumabtreated patients who achieved 2-h pain freedom was $13.8(-17.1,-10.5 ; n=54)$ compared with -4.9 in patients receiving placebo who were pain free at $2 \mathrm{~h}(-9.1$, -0.6 ; $n=29$; Fig. 2A). Among 2 -h pain freedom responders (eptinezumab, 56/238; placebo, 29/242), a larger proportion of the eptinezumab group (37.5\%) did not experience a new migraine attack within the 4-week follow-up period of the study compared with patients in the placebo group (17.9\%) (Fig. 2B). Similar benefits of early response were observed in patients experiencing absence of (MBS at $2 \mathrm{~h}$, headache pain freedom at $4 \mathrm{~h}$, and absence of MBS at $4 \mathrm{~h}$ (see Additional file 1, Table 3).

\section{6-item Migraine Treatment Optimization Questionnaire}

At baseline, the mTOQ- 6 total score was similar across both treatment arms (eptinezumab, 18.1; placebo, 18.6) and reflective of patients diagnosed with episodic migraine [21]. At week 4, the LS mean $(95 \% \mathrm{CI})$ change from baseline in mTOQ-6 total score (Fig. 3A) was 2.1 $(1.5,2.6)$ in patients treated with eptinezumab, compared with $1.2(0.7,1.7)$ in patients receiving placebo $(P<.01)$. For patients reporting headache pain freedom

Table 1 Overview of demographics and baseline headache characteristics

\begin{tabular}{|c|c|c|}
\hline & $\begin{array}{l}\text { Eptinezumab } \\
(N=238)\end{array}$ & $\begin{array}{l}\text { Placebo } \\
(N=242)\end{array}$ \\
\hline Age (years), mean (SD) & $44.9(12.0)$ & $44.1(12.1)$ \\
\hline Sex: Female, no. (\%) & $202(84.9)$ & $201(83.1)$ \\
\hline \multicolumn{3}{|l|}{ Race, no. (\%) } \\
\hline White & $200(84.0)$ & $213(88.0)$ \\
\hline Black or African American & $30(12.6)$ & $19(7.9)$ \\
\hline Other $^{a}$ & $8(3.4)$ & $10(4.1)$ \\
\hline History of chronic migraine, no. $(\%)^{b}$ & $25(10.5)$ & $27(11.2)$ \\
\hline Monthly migraine days, mean $(S D)^{c}$ & $7.2(2.7)$ & $7.2(2.6)$ \\
\hline Duration of migraine prior to infusion start (hours), mean (SD) ${ }^{d}$ & $3.7(1.0)$ & $3.7(1.0)$ \\
\hline \multicolumn{3}{|l|}{ Severity of headache pain, no. (\%) } \\
\hline Moderate & $110(46.2)$ & $117(48.3)$ \\
\hline Severe & $128(53.8)$ & $123(50.8)$ \\
\hline
\end{tabular}

SD standard deviation

${ }^{a}$ Other includes Asian, American Indian or Alaska Native, Native Hawaiian or other Pacific Islander, other, and multiple

${ }^{\mathrm{b}}$ Migraine history was collected at the screening visit by the investigator through medical records; if medical records could not be obtained, history was

confirmed via patient interview in order to obtain sufficient information to confirm all eligibility criteria are met

'Patients self-reported the average number of monthly migraine days over the 3 months prior to screening

${ }^{\mathrm{d} D u r a t i o n}$ was calculated as the difference between the study drug infusion start date and time and the day 1 headache start date and time 
Table 2 Patient-reported outcomes at baseline and week 4

\begin{tabular}{|c|c|c|}
\hline & Eptinezumab & Placebo \\
\hline HIT-6 total score, $n^{a}$ & 226 & 232 \\
\hline Baseline, mean (SD) & $65.1(4.97)$ & $64.8(5.01)$ \\
\hline Week 4, mean (SD) & $57.0(9.74)$ & $61.1(7.83)$ \\
\hline Change from baseline, LS mean ( $95 \%$ Cl) & $-8.7(-10.1,-7.3)$ & $-4.5(-5.9,-3.1)$ \\
\hline Difference from placebo, LS mean $(95 \% \mathrm{CI})^{\mathrm{b}}$ & $-4.2(-5.7,-2.6)$ & \\
\hline$P$-value vs placebo & $<.0001$ & \\
\hline mTOQ-6 total score, $n^{a}$ & 226 & 231 \\
\hline Baseline, mean (SD) & $18.1(4.05)$ & $18.6(4.20)$ \\
\hline Week 4, mean (SD) & $20.1(4.21)$ & $19.6(4.22)$ \\
\hline Change from baseline, LS mean (95\% CI) & $2.1(1.5,2.6)$ & $1.2(0.7,1.7)$ \\
\hline Difference from placebo, LS mean $(95 \% \mathrm{Cl})^{\mathrm{b}}$ & $0.9(0.3,1.5)$ & \\
\hline$P$-value vs placebo & .0053 & \\
\hline PGIC rating at week $4, n^{a}$ & 226 & 232 \\
\hline Very much improved, n (\%) & $56(24.8)$ & $29(12.5)$ \\
\hline Much improved, n (\%) & $78(34.5)$ & $31(13.4)$ \\
\hline Minimally improved, n (\%) & $54(23.9)$ & $58(25.0)$ \\
\hline No change, n (\%) & $35(15.5)$ & $109(47.0)$ \\
\hline Worse, $\mathrm{n}(\%)^{c}$ & $3(1.3)$ & $5(2.1)$ \\
\hline
\end{tabular}

Cl confidence interval, HIT-6 6-item Headache Impact Test, LS least squares, mTOQ-6 6-item Migraine Treatment Optimization Questionnaire, PGIC Patient Global Impression of Change, SD standard deviation

a Limited to patients with both baseline and post-baseline data. All $P$-values are descriptive

${ }^{b}$ The estimated mean, mean difference from placebo, and $95 \% \mathrm{Cl}$ are from an analysis of covariance adjusted for baseline value and stratification factors of concomitant treatment and region

"Worse includes "minimally worse", "much worse", and "very much worse"

at $2 \mathrm{~h}$ post infusion start, the improvement in mTOQ-6 was $3.1(1.7,4.5)$ in patients treated with eptinezumab, compared with $1.8(0.5,3.2)$ in patients receiving placebo. Analysis of mTOQ-6 item scores was again similar across treatment groups at baseline (see Additional file 1, Table 4), with analysis of the change from baseline at week 4 resulting in greater improvements for mTOQ-6 item 1 (quickly return to function) and item 2 (pain-free within $2 \mathrm{~h})$ when compared with placebo $(P<.01)$ (Fig. 3B).

\section{Patient Global Impression of Change}

Analysis of the PGIC (Table 2) identified more than twice as many eptinezumab-treated patients reporting "much" or "very much" improvement in disease status compared with placebo ( $59.3 \%$ vs $25.9 \%$, respectively). Overall, clinical improvement was reported by $83.2 \%$ of eptinezumab-treated patients compared with $50.9 \%$ of placebo patients. No change in disease status was reported by $15.5 \%$ of eptinezumab-treated patients compared with $47.0 \%$ of placebo patients.

\section{Discussion}

In the RELIEF study, patients receiving eptinezumab, a preventive migraine treatment, during a migraine attack achieved a faster time to headache pain freedom and absence of MBS compared with patients receiving placebo [19]. Patients with migraine who were administered eptinezumab during a migraine attack demonstrated greater improvements in the PROMs of HIT-6, mTOQ-6, and PGIC compared to those receiving placebo.

We also compared the benefits of treatment on these PROMs among those who did and did not achieve freedom from pain and MBS $2 \mathrm{~h}$ post treatment. A favorable short-term (2-h) response to treatment was associated with a favorable response to PROMs at 4 weeks. This short-term response, which occurred more often in eptinezumab-treated patients, resulted in the reduction of overall headache-related impact (HIT-6) as well as a reduction in the potential for experiencing another migraine attack during the study period. For patients with frequent migraine attacks (4-15 days per month), experiencing both early (2-h) onset of treatment effect and a decreased chance of having another migraine attack for the 4 weeks following infusion could underly the robust improvement observed on the HIT-6 in this population. Prior work has not evaluated the association between acute benefits of a preventive treatment and longer-term benefits; further research is needed to determine if acute treatment success for a single attack might reduce 
A.

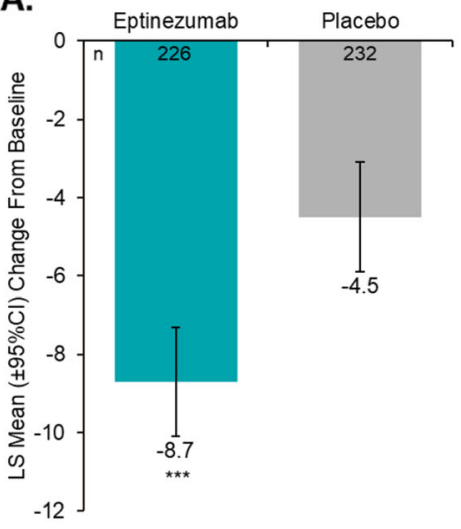

B.

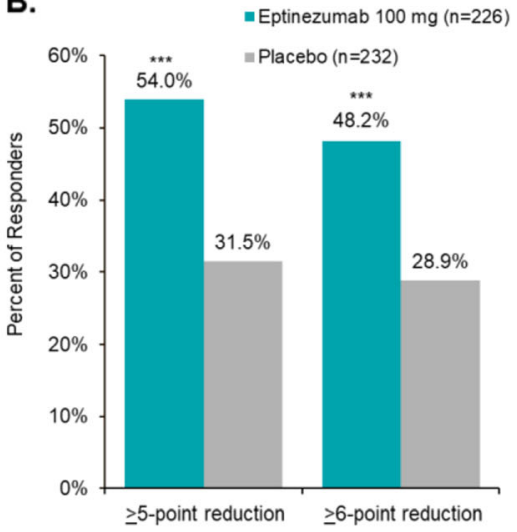

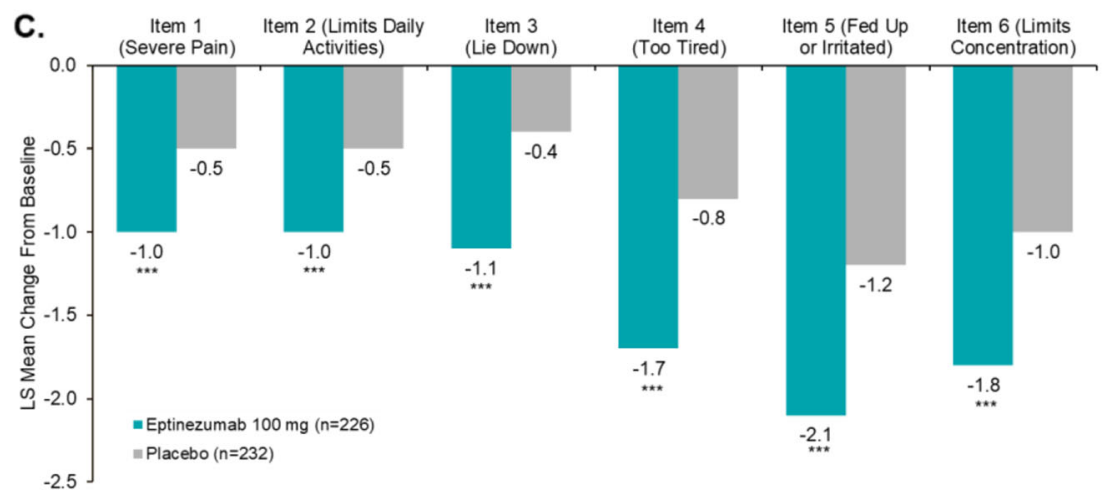

D.

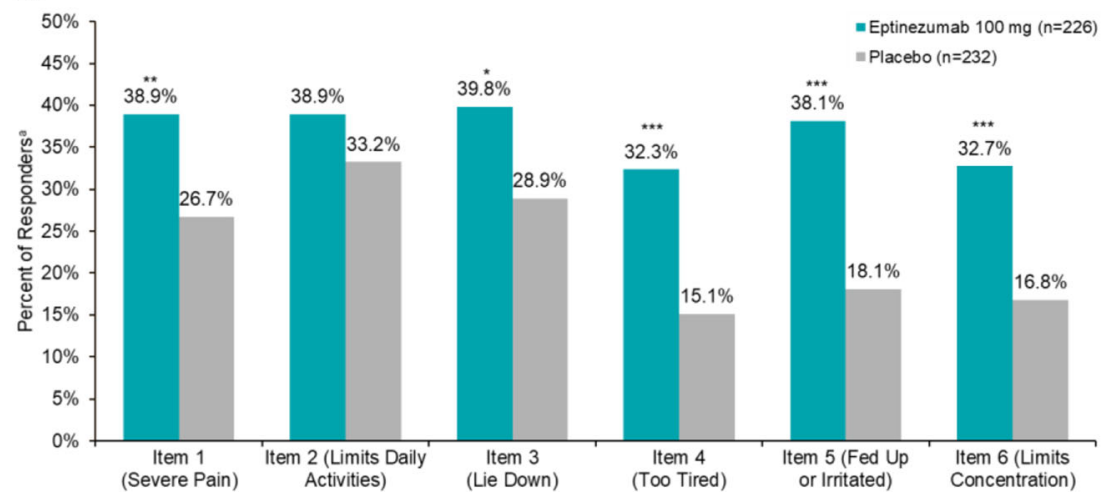

Fig. 1 Effects of Eptinezumab vs Placebo on HIT-6-Related Outcomes. A Least Squares (LS) Mean Change From Baseline at Week 4; B Responder Rates at Week 4 for HIT-6 Total Score; C LS Mean Change From Baseline in HIT-6 Item Scores at Week 4; D Responder Rates for HIT-6 Item Scores at Week 4. Cl, confidence interval; HIT-6, 6-item Headache Impact Test; LS, least square. ${ }^{*} P<.05$, ${ }^{*} P<.01$, ${ }^{* * *} P<.001$ vs placebo. Limited to patients with both baseline and post-baseline HIT-6 data. ${ }^{a}$ For items $1-3$, a responder was defined as a patient with an improvement of $\geq 1$ category; for items 4-6, a responder was defined as a patient with an improvement of $\geq 2$ categories [24]. Error bars represent $95 \% \mathrm{Cl}$

headache frequency on a longer term basis. It should be noted that, as a preventive treatment, eptinezumab is administered every 12 weeks, with a previous analysis showing statistically significant preventive efficacy relative to placebo beginning on post-treatment day 1 and extending through the 12-week dosing interval [26]; therefore, some of the effect on week 4 outcomes was likely related to the rapid onset of preventive efficacy with eptinezumab.

These findings confirm the substantial burden that migraine places on patients and identified a treatment benefit in favor of eptinezumab compared with placebo in relieving the disease burden in patients experiencing up to 15 migraine days per month. This treatment 


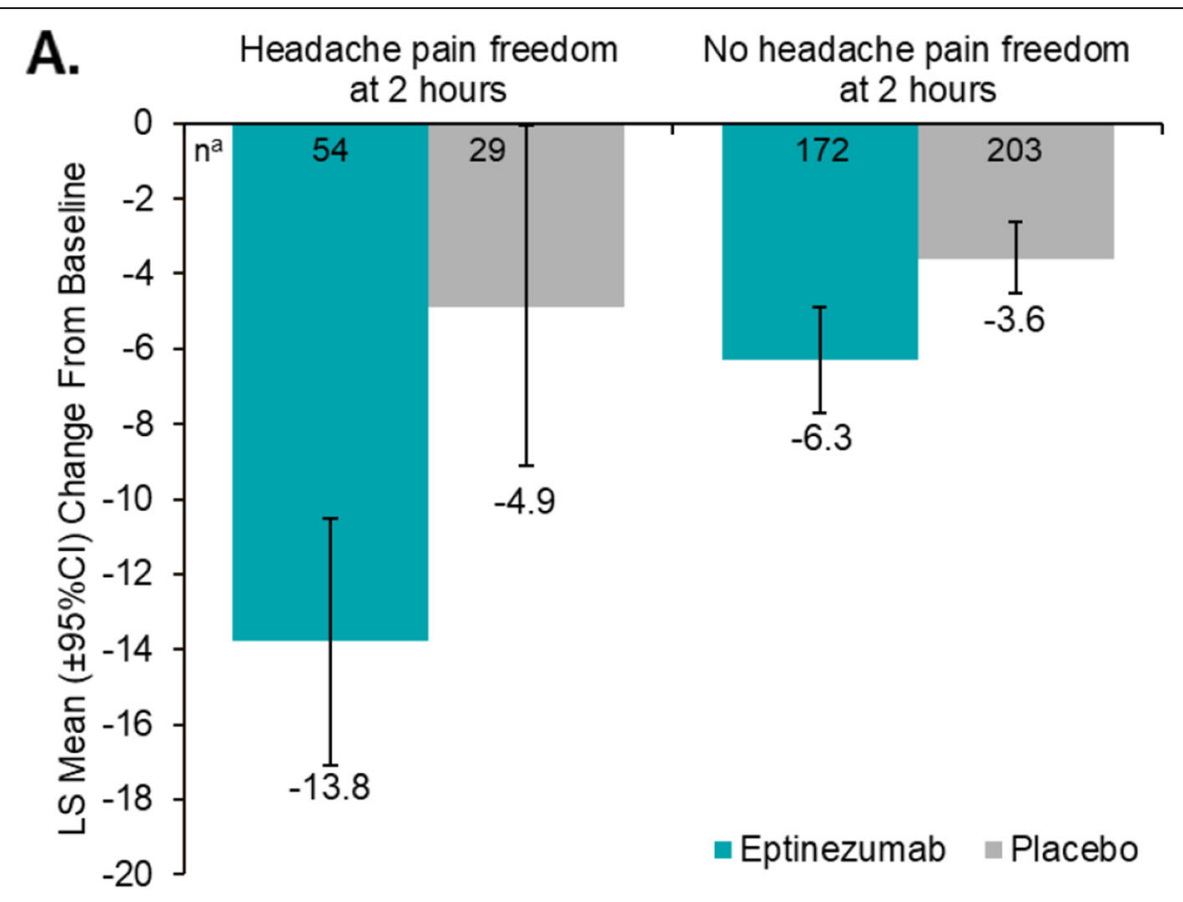

B.

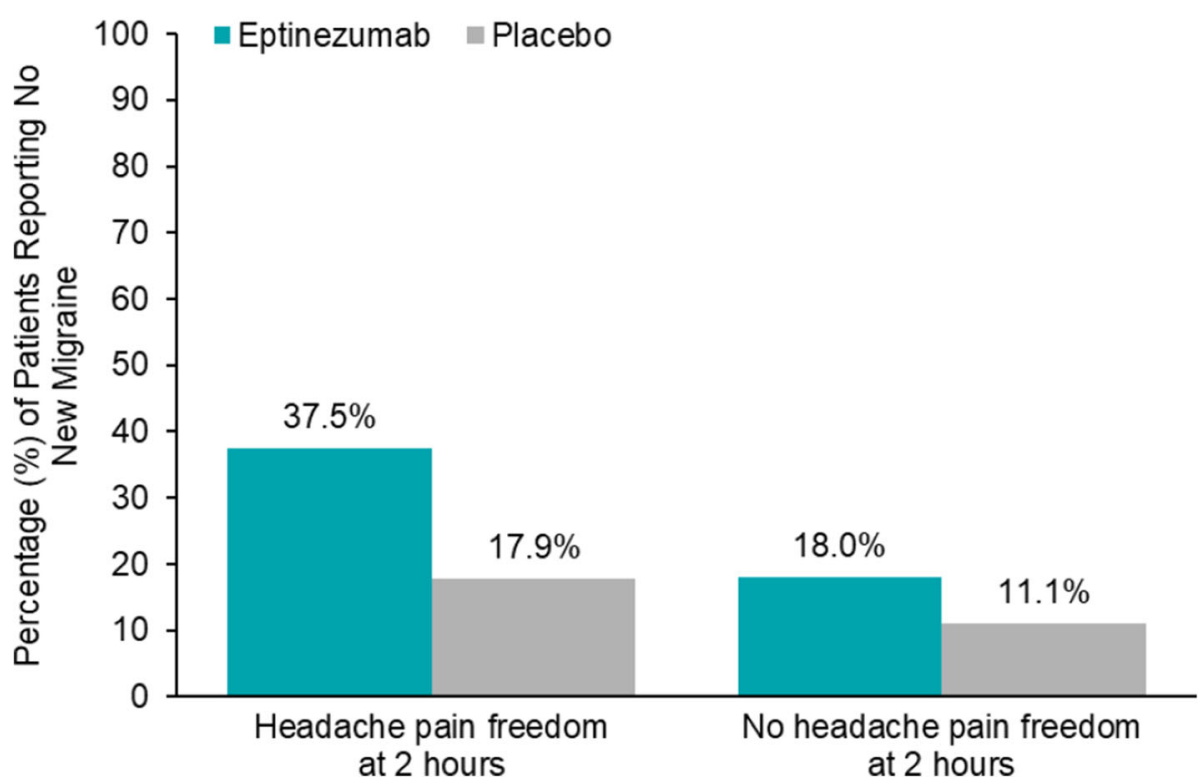

Fig. 2 Relationship of 2-h Pain Freedom to Change in HIT-6 and Occurrence of Subsequent Migraine. A Mean Change From Baseline to Week 4 in HIT-6 Total Score and B Percent of Patients Without a New Migraine Occurring With or Without Headache Pain Freedom at 2 Hours After Infusion Start. Cl, confidence interval; HIT-6, 6-item Headache Impact Test; LS, least square. "Limited to patients with both baseline and postbaseline HIT-6 data

Error bars represent $95 \% \mathrm{Cl}$

benefit, as demonstrated by improvements in HIT-6 (in the total population as well as the more pronounced improvements in patients with headache pain freedom $2 \mathrm{~h}$ after start of eptinezumab infusion), was clinically meaningful according to the guidance from the AHS [23] and meets the criteria established for a clinically meaningful change in patients with chronic migraine at a total score and individual item level [24]. Further, the patientreported treatment benefits observed on the HIT- 6 in this study replicate the results reported in the phase 3 PROMISE-2 study in patients with chronic migraine [18, 27], underpinning the importance of evaluating and 


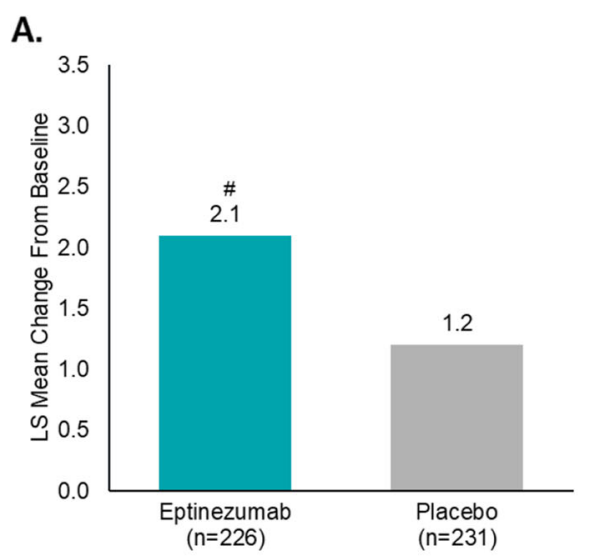

B.

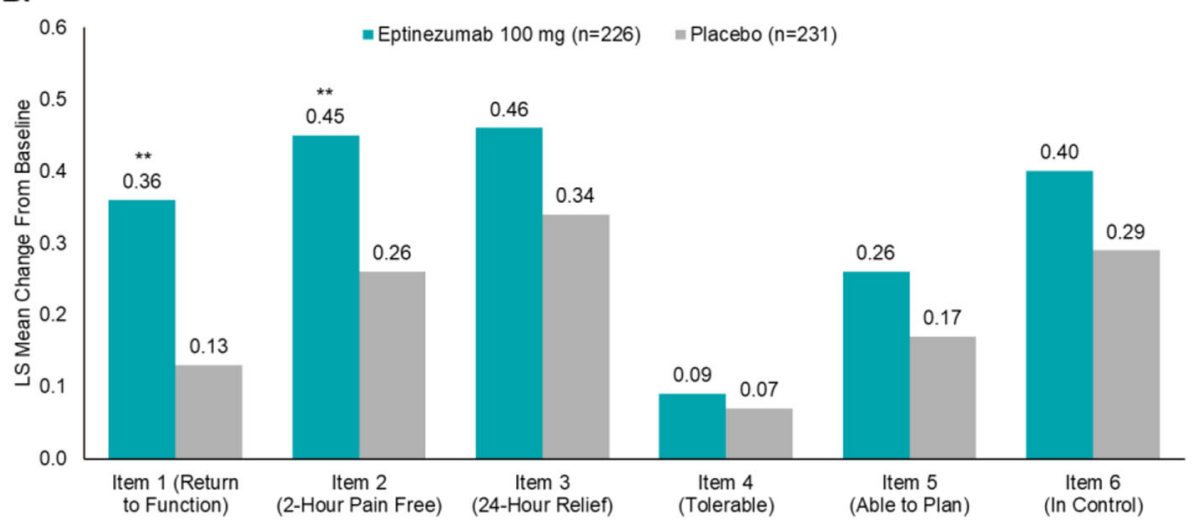

Fig. 3 mTOQ-6 Mean Change From Baseline to Week 4. A Mean change from baseline to week 4 in mTOQ-6 total score and $\mathbf{B}$ in mTOQ-6 item scores. mTOQ-6, 6-item Migraine Treatment Optimization Questionnaire. 'Limited to patients with both baseline and post-baseline mTOQ-6 data. ${ }^{* *} P<.01, \# P=.0053$ vs placebo

monitoring the impact of migraine with the HIT-6 in all patients.

The impact of eptinezumab treatment on the PGIC highlights the importance of evaluating the patient's perception of benefits that are directly associated with treatment. The present analyses identified that more than twice as many patients treated with eptinezumab reported "much improved" or "very much improved" on the PGIC compared to placebo, indicating a high degree of patient satisfaction associated with eptinezumab. Studies in migraine patients have found a direct correlation between PGIC improvement and a reduction in impact on the HIT-6 [24], and while not tested in this patient population, it is expected that similar results would be observed, further supporting the clinical meaningfulness of the PGIC results. Of particular interest is the greater number of patients indicating "much improved" or "very much improved" for those who reported headache pain freedom at $2 \mathrm{~h}$. In this population, the PGIC response at week 4 highlights the importance of an early "patient-perceived" response to treatment in the long-term management of migraine.
Patients responded better on mTOQ-6 at 4 weeks after treatment with eptinezumab compared to those who received placebo, based on change from baseline in mTOQ-6 total score, suggesting that eptinezumab may work synergistically with acute treatments used to treat future migraines. Patients treated with eptinezumab reported a better score compared to patients receiving placebo on mTOQ-6 items 1 and 2 (return to normal and pain free within $2 \mathrm{~h}$ ), indicating they were more likely to achieve 2-h pain-free outcomes when treating future migraine attacks and able to function better. The lack of difference between eptinezumab-treated and placebo patients on items 5 and 6 (planning activities and expecting no disruptions due to migraine) indicates that although patients report favorably on returning to normal, it may take longer than 4 weeks for patients to feel in control of managing their migraine, especially given that most patients were experiencing fewer migraine attacks at week 4 than they did at baseline. Given patients were administered eptinezumab in an acute treatment setting, they had no expectations that their usual acute medication usage would be impacted; therefore, findings 
that eptinezumab provided a greater ability to return to normal functioning (item 1), be pain-free at $2 \mathrm{~h}$ after acute treatment (item 2), and had no impact on the tolerability of current medication (item 4) are consistent with a potential of eptinezumab to work synergistically with acute migraine treatments.

\section{Limitations}

Several limitations should be considered. The data for the RELIEF study were generated in the clinical trial setting; thus, participants may differ from those in general practice, potentially limiting the overall generalizability of the results. Further, patients reported a higher migraine severity and impact than previously reported in similar patient populations. Additionally, because changes in MMDs-a traditional outcome for migraine preventive treatments-were not captured over longer term (e.g. over 12 weeks) using a daily electronic diary, changes in PROMs could not be compared to reductions in MMDs. Finally, while PROM assessment demonstrated a clinically meaningful benefit at week 4, no PROMs were captured after 12 weeks (the approved treatment interval for eptinezumab), limiting the longterm applicability of the results since it likely takes longer than 4 weeks for patients to adjust their expectations after initiation of preventive treatment.

\section{Conclusions}

Preventive migraine treatment with eptinezumab initiated during a migraine attack provided clinically meaningful improvements in several PROMs as early as 4 weeks after infusion. These benefits were especially noticeable in those patients who reported headache pain freedom at $2 \mathrm{~h}$, highlighting the significance of experiencing the early onset of effect with eptinezumab. In addition, patients treated with eptinezumab relative to those receiving placebo reported greater effectiveness of their acute medication used after initiation during a migraine attack, findings that may raise the standards for preventive treatments and provide promising outcomes for patients suffering from migraine.

\section{Supplementary Information}

The online version contains supplementary material available at https://doi. org/10.1186/s10194-021-01376-7.

\section{Additional file 1.}

\section{Acknowledgements}

The authors thank the patients, their families, and the study sites that participated in this study. The authors also thank Nicole Coolbaugh, CMPP, and Philip Sjostedt, PharmD, of The Medicine Group, LLC (New Hope, PA, USA) for providing medical writing support, which was funded by $\mathrm{H}$. Lundbeck A/S (Copenhagen, Denmark) in accordance with Good Publication Practice guidelines.

\section{Role of the funder/sponsor}

In collaboration with the academic authors, the sponsor participated in the design and conduct of the study and in the collection, management, analysis, and interpretation of the data. The preparation, review, and approval of the manuscript was undertaken by all authors and by a professional medical writer and editor funded by the sponsor. Most statistical analyses were performed by a contracted research organization and were directed under contractual agreement with $\mathrm{H}$. Lundbeck A/S (Copenhagen, Denmark); additional analyses were performed by Annika Lindsten, an employee of $\mathrm{H}$. Lundbeck A/S. All authors and H. Lundbeck A/S prepared, reviewed, and approved the final version of the manuscript and made the decision to submit the manuscript for publication. The sponsor did not have the right to veto publication or to control the decision regarding to which journal the paper was submitted.

\section{Authors' contributions}

PKW had full access to all data in the study and takes responsibility for the integrity of the data and the accuracy of the data analysis. RC and LM established the concept and design of the manuscript. All authors contributed to the acquisition, analysis, interpretation of data, and drafting and revision of the manuscript. MKJ and AL performed the statistical analysis. PKW, PM, GC, JA, DCB, and RBL obtained funding. MKJ, AE, and RC offered administrative support and supervision for this project. The author(s) read and approved the final manuscript.

\section{Funding}

The study was sponsored and funded by $\mathrm{H}$. Lundbeck A/S (Copenhagen, Denmark). Editorial support for the development of this manuscript was funded by $\mathrm{H}$. Lundbeck $\mathrm{A} / \mathrm{S}$.

\section{Availability of data and materials}

In accordance with EFPIA's and PhRMA's "Principles for Responsible Clinical Trial Data Sharing" guidelines, Lundbeck is committed to responsible sharing of clinical trial data in a manner that is consistent with safeguarding the privacy of patients, respecting the integrity of national regulatory systems, and protecting the intellectual property of the sponsor. The protection of intellectual property ensures continued research and innovation in the pharmaceutical industry.

The data were collected during the study using an electronic data capture (EDC) device and reported on each patient's individual case report form (CRF). All data reports, including the PGIC, HIT-6, and mTOQ-6 questionnaires, were documented within the EDC system, with each set of completed CRFs reviewed and verified by the site monitor and electronically signed and dated by the investigator. Deidentified data are available to those whose request has been reviewed and approved through an application submitted to https://www.lundbeck.com/global/our-science/clinical-data-sharing

\section{Declarations}

Ethics approval and consent to participate

Approval for each study was provided by the independent ethics committee or institutional review board of the study sites. The studies were each conducted in accordance with Good Clinical Practice guidelines, the principles of the Declaration of Helsinki, and all applicable regulatory requirements. Patients provided written informed consent prior to initiation of any study procedures.

Consent for publication

Not applicable.

\section{Competing interests}

PM reported receiving personal fees and research support from Abbvie, Amgen/Novartis, Biohaven, Lilly, Lundbeck, and Teva.

PKW reported receiving consulting fees from Abbvie, Amgen, Biohaven, Lilly, Lundbeck, Novartis, Teva; serving on speaker bureaus for Abbvie, Amgen, Biohaven, Lilly, Lundbeck, Novartis, Teva; and receiving research support from Abbvie, Amgen, AZ, Biogen, Lilly, Lundbeck, Novartis, Supernus, Teva. JA reported receiving clinical trial grants from Abbvie, Biohaven, Lilly, Satsuma, and Zosano; consulting fees from Abbvie, Amgen, Axsome, Biohaven, electroCore, Impel, Lilly, Lundbeck, Revance, Satsuma, Theranica, 
and Vorso; and speaker fees from Abbvie, Amgen, Biohaven, Impel, Lilly, Lundbeck, and Satsuma.

DCB has received grant support from Amgen, the National Headache Foundation, and the FDA; has received consulting support from Allergan/ Abbvie, Amgen, Lilly, Lundbeck, and Teva; and serves on the editorial board of Current Pain and Headache Reports.

RBL receives or has received, as a consultant and/or advisory panel member, honoraria from Lundbeck Seattle BioPharmaceuticals, Allergan, American Academy of Neurology, American Headache Society, Amgen, Biohaven Pharmaceuticals, BioVision, Boston Scientific, Dr. Reddy's Laboratories, electroCore Medical, eNeura Therapeutics, GlaxoSmithKline, Lilly, Merck, Pernix, Pfizer, Supernus, Teva Pharmaceuticals, Trigemina, Vector, and Vedanta; received compensation from eNeura and Biohaven Pharmaceuticals, has stock or stock options in Biohaven Pharmaceuticals and Manistee; receives research support from Amgen, Migraine Research Foundation, and National Headache Foundation.

GC had nothing to declare.

$\mathrm{MKJ}, \mathrm{AL}$, and $\mathrm{AE}$ are employees of Lundbeck or one of its subsidiary companies.

LM was an employee of Lundbeck Seattle BioPharmaceuticals at the time of study. RC was an employee of Lundbeck or one of its subsidiary companies at the time of study and manuscript development.

\section{Author details}

${ }^{1}$ New England Institute for Neurology and Headache, Stamford, CT, USA. ${ }^{2}$ Palm Beach Headache Center, West Palm Beach, FL, USA. ${ }^{3}$ Neurology Research Institute Palm Beach, West Palm Beach, FL, USA. " Premiere Research Institute, West Palm Beach, FL, USA. ${ }^{5}$ Palm Beach Neurology, West Palm Beach, FL, USA. ${ }^{6}$ Nova Southeastern University, Fort Lauderdale, FL, USA. ${ }^{7}$ Department of Neurology, Georgetown University Hospital, Washington, DC, USA. ${ }^{8}$ Albert Einstein College of Medicine, Bronx, NY, USA. ${ }^{9}$ Vector Psychometric Group, LLC, Chapel Hill, NC, USA. ${ }^{0}$ Multiprofile Clinic Consilium Medulla, Georgian Association of Medical Specialties, David Tvildiani Medical University, Tbilisi, Georgia. ${ }^{11} \mathrm{H}$. Lundbeck A/S, Copenhagen, Denmark. ${ }^{12}$ Lundbeck Seattle BioPharmaceuticals, Inc., Bothell, WA, USA. ${ }^{13}$ Lundbeck LLC, Deerfield, IL, USA.

Received: 11 November 2021 Accepted: 20 December 2021 Published online: 07 February 2022

\section{References}

1. Headache Classification Committee of the International Headache Society (IHS) (2018) The international classification of headache disorders, 3rd edition. Cephalalgia. 38(1):1-211. https://doi.org/10.1177/0333102417738202

2. Buse DC, Fanning KM, Reed ML, Murray S, Dumas PK, Adams AM et al (2019) Life with migraine: effects on relationships, career, and finances from the Chronic Migraine Epidemiology and Outcomes (CaMEO) study. Headache. 59(8):1286-1299. https://doi.org/10.1111/head.13613

3. Lipton RB, Bigal ME, Diamond M, Freitag F, Reed ML, Stewart WF (2007) Migraine prevalence, disease burden, and the need for preventive therapy. Neurology. 68(5):343-349. https://doi.org/10.1212/01.wnl.0000252808.9764 9.21

4. Munakata J, Hazard E, Serrano D, Klingman D, Rupnow MF, Tierce J et al (2009) Economic burden of transformed migraine: results from the American Migraine Prevalence and Prevention (AMPP) study 1. Headache. 49(4):498-508

5. Blumenfeld AM, Varon SF, Wilcox TK, Buse DC, Kawata AK, Manack A et al (2011) Disability, HRQoL and resource use among chronic and episodic migraineurs: results from the International Burden of Migraine Study (IBMS). Cephalalgia. 31(3):301-315. https://doi.org/10.1177/0333102410381145

6. Martelletti P, Schwedt TJ, Lanteri-Minet M, Quintana R, Carboni V, Diener HC et al (2018) My migraine voice survey: a global study of disease burden among individuals with migraine for whom preventive treatments have failed. J Headache Pain 19(1):1 15. https://doi.org/10.1186/s10194-018-0946-z

7. Buse DC, Scher Al, Dodick DW, Reed ML, Fanning KM, Manack Adams A et al (2016) Impact of migraine on the family: perspectives of people with migraine and their spouse/domestic partner in the CaMEO study. Mayo Clin Proc 91(5):596-611. https://doi.org/10.1016/j.mayocp.2016.02.013

8. Lipton RB, Stewart WF, Diamond S, Diamond ML, Reed M (2001) Prevalence and burden of migraine in the United States: data from the American migraine study II. Headache. 41(7):646-657
9. Katsarava Z, Buse DC, Manack AN, Lipton RB (2012) Defining the differences between episodic migraine and chronic migraine. Curr Pain Headache Rep 16(1):86-92. https://doi.org/10.1007/s11916-011-0233-z

10. Lipton RB, Silberstein SD (2015) Episodic and chronic migraine headache: breaking down barriers to optimal treatment and prevention. Headache. 55(Suppl 2):103-122; quiz 123-106. https://doi.org/10.1111/head.12505_2

11. Weatherall MW (2015) The diagnosis and treatment of chronic migraine. Ther Adv Chronic Dis 6(3):115-123. https://doi.org/10.1177/204062231 5579627

12. Marmura MJ (2018) Triggers, protectors, and predictors in episodic migraine. Curr Pain Headache Rep 22(12):81. https:/doi.org/10.1007/s11916-018-0734-0

13. MCGinley JS, Houts CR, Nishida TK, Buse DC, Lipton RB, Goadsby PJ et al (2021) Systematic review of outcomes and endpoints in preventive migraine clinical trials. Headache. 61(2):253-262. https://doi.org/10.1111/head.14069

14. Houts CR, McGinley JS, Nishida TK, Buse DC, Wirth RJ, Dodick DW et al (2021) Systematic review of outcomes and endpoints in acute migraine clinical trials. Headache. 61(2):263-275 https://doi.org/10.1111/head.14067

15. WEPTI [package insert]. Bothell: Lundbeck Seattle BioPharmaceuticals, Inc.; 2021

16. Garcia-Martinez LF, Raport CJ, Ojala EW, Dutzar B, Anderson K, Stewart E et al (2020) Pharmacologic characterization of ALD403, a potent neutralizing humanized monoclonal antibody against the calcitonin gene-related peptide. JPET. 374(1):93-103. https://doi.org/10.1124/jpet.119.264671

17. Ashina M, Saper J, Cady R, Schaeffler B, Biondi DM, Hirman J et al (2020) Eptinezumab in episodic migraine: a randomized, double-blind, placebocontrolled study (PROMISE-1). Cephalalgia. 40(3):241-254. https://doi.org/1 $0.1177 / 0333102420905132$

18. Lipton RB, Goadsby PJ, Smith J, Schaeffler BA, Biondi DM, Hirman J et al (2020) Efficacy and safety of eptinezumab in patients with chronic migraine. PROMISE-2. Neurology. 94(13):e1365-e1377. https://doi.org/10.1212/WNL. 0000000000009169

19. Winner PK, McAllister P, Chakhava G, Ailani J, Ettrup A, Krog Josiassen M et al (2021) Effects of intravenous eptinezumab vs placebo on headache pain and most bothersome symptom when initiated during a migraine attack: a randomized clinical trial. JAMA. 325(23):2348-2356. https://doi.org/1 0.1001/jama.2021.7665

20. Kosinski M, Bayliss MS, Bjorner JB, Ware JE Jr, Garber WH, Batenhorst A et al (2003) A six-item short-form survey for measuring headache impact: the HIT-6. Qual Life Res 12(8):963-974. https://doi.org/10.1023/A:1026119331193

21. Lipton RB, Kolodner K, Bigal ME, Valade D, Lainez MJ, Pascual J et al (2009) Validity and reliability of the migraine-treatment optimization questionnaire. Cephalalgia. 29(7):751-759. https://doi.org/10.1111/j.1468-2982.2008.01786.x

22. Guy W. ECDEU Assessment Manual for Psychopharmacology. U.S. Department of Health, Education, and Welfare, Public Health Service, Alcohol, Drug Abuse, and Mental Health Administration, National Institute of Mental Health, Psychopharmacology Research Branch, Division of Extramural Research Programs. 1976

23. American Headache Society (2019) The American Headache Society position statement on integrating new migraine treatments into clinical practice. Headache. 59(1):1-18. https://doi.org/10.1111/head.13456

24. Houts CR, Wirth RJ, McGinley JS, Cady R, Lipton RB (2020) Determining thresholds for meaningful change for the Headache Impact Test (HIT-6) total and item-specific scores in chronic migraine. Headache. 60(9):20032013. https://doi.org/10.1111/head.13946

25. Houts CR, McGinley JS, Wirth RJ, Cady R, Lipton RB (2021) Reliability and validity of the 6-item Headache Impact Test in chronic migraine from the PROMISE-2 study. Qual Life Res 30:931-943. https://doi.org/10.1007/s11136020-02668-2

26. Dodick DW, Gottschalk C, Cady R, Hirman J, Smith J, Snapinn S (2020) Eptinezumab demonstrated efficacy in sustained prevention of episodic and chronic migraine beginning on day 1 after dosing. Headache. 60(10):22202231 https://doi.org/10.1111/head.14007

27. Silberstein S, Diamond M, Hindiyeh NA, Biondi DM, Cady R, Hirman J et al (2020) Eptinezumab for the prevention of chronic migraine: efficacy and safety through 24 weeks of treatment in the phase 3 PROMISE-2 (prevention of migraine via intravenous ALD403 safety and efficacy-2) study. J Headache Pain 21(1):120. https://doi.org/10.1186/s10194-020-011 86-3

\section{Publisher's Note}

Springer Nature remains neutral with regard to jurisdictional claims in published maps and institutional affiliations. 